\title{
Life-threatening Thrombotic Complications in Pregnancy
}

\section{Sunita Kumar}

\begin{abstract}
Aim: Thrombotic complications like thrombotic thrombocytopenic purpura (TTP), hemolytic uremic syndrome (HUS), and posterior reversible encephalopathy syndrome (PRES) in pregnancy are due to the deposits of platelet and fibrin in the microvasculature damaging and destroying the passing red blood cells (RBCs) and platelets so as to cause hemolysis, thrombocytopenia, and anemia. They are relatively rare fulminant life-threatening conditions, which can lead to significant perinatal and maternal morbidity and/or mortality. Pregnancy itself is thrombus prone and conditions like preeclamptic toxemia (PET), eclampsia, and hemolysis, elevated liver enzymes, and low platelet count (HELLP) are not only occasionally associated, but can add to the gravity of the seriousness.
\end{abstract}

Case report: Three cases each of TTP, HUS, and PRES are being presented with their case history treatment and outcome to show the similarity in their presentation along with diagnostic dilemma, clinching diagnostic tests, and overall management with specific treatment in individual cases.

Conclusion: The TTP, HUS, and PRES are life-threatening complications in pregnancy without any signs of early recognition. Awareness of the condition and prompt action is the need to avoid perinatal and maternal mortality and morbidity associated with these syndromes.

Keywords: Hemolytic uremic syndrome, Life-threatening thrombotic complications, Pregnancy Thrombotic complications in pregnancy, Thrombotic thrombocytopenic purpura.

How to cite this article: Kumar S. Life-threatening Thrombotic Complications in Pregnancy. J South Asian Feder Obst Gynae 2018;10(2):138-141.

Source of support: Nil

Conflict of interest: None

Date of received: 15 March 2018

Date of acceptance: 20 April 2018

Date of publication: July 2018

\section{INTRODUCTION}

Thrombotic thrombocytopenic purpura and HUS involve nonimmunologic platelet destruction. Loose strands of platelet and fibrin are deposited in multiple small vessels

Senior Consultant

Department of Obstetrics and Gynecology, Sir Ganga Ram City Hospital, New Delhi, India

Corresponding Author: Sunita Kumar, Senior Consultant Department of Obstetrics and Gynecology, Sir Ganga Ram City Hospital, New Delhi, India, e-mail: drsunitakumar@hotmail.com and damage passing platelets and $\mathrm{RBC}$, causing significant thrombocytopenia and anemia. ${ }^{1}$ Pregnancy-associated thrombotic microangiopathy (P-TMA) is a rare disorder with an estimated incidence of approximately 1:25,000 pregnancies; however, it is associated with significant perinatal and/or maternal morbidity and mortality.

Pregnancy is associated with a lot of physiological changes in the women, involving the functioning of various organs and the blood becoming more viscous and thrombus prone. Hypertension in pregnancy is a common complication and is often associated with preeclampsia, eclampsia, and HELLP syndrome along with thrombotic changes and its consequences in the mother and the growing fetus.

Thrombotic thrombocytopenic purpura, HUS, and PRES are important life-threatening thrombotic complications which are not only fulminant and life-threatening but are devoid of any sings for an early recognition, surprising the obstetrician with the sudden onset of distressing signs and symptoms in an otherwise supposed to be normal patient. Such patients have multiorgan involvement requiring a multidisciplinary approach, but the onus still lies with the obstetrician to manage the patient so as to have a timely delivery depending upon the gestational age, fetal condition, and the most important, clinical response of the patient to the treatment.

Three cases of TTP, HUS, and PRES each are being presented to stress upon the similar abrupt fulminant clinical course, overall treatment, but distinguishing features on lab reports for TTP, HUS, PRES, and HELLP, all of which may at times require specific treatment in individual cases.

\section{CASE REPORTS}

\section{Case 1}

A young primipara at 38 weeks of pregnancy presented with high blood pressure and facial palsy on the right side. She was treated with corticosteroids and antihypertensive medicines. Her blood investigations showed raised liver enzymes with low platelet count. Diagnostic dilemma of TTP and HELLP existed. Her blood pressure and facial paralysis as well as blood reports improved with treatment before the delivery. She had a cesarean section for nonprogress and gave birth to a healthy baby, but did not require any plasma exchange or any other specific treatment. In view of her blood reports, 
symptoms, and improvement with treatment, she was labeled to have TTP.

It is vital to start the treatment as early as possible without any delay, as certain specific blood tests which could clinch the diagnosis may take time to be reported.

\section{Case 2}

A 34-year-old lady with an in vitro fertilization pregnancy and twins was referred with preterm contractions and high blood pressure at 28 weeks of pregnancy. She was clinically anemic and her investigations showed an hemoglobin of $8 \mathrm{gm} \%$, but normal liver and kidney function tests (KFT). On detailed history, she had diarrhea with bloody stools 15 days back and since then, she developed anemia.

She was treated with antihypertensive, iron, and duvadilon drip but she became restless all of a sudden the next day in the evening. Her blood pressure was 150/100 $\mathrm{mm} \mathrm{Hg}$, face was flushed, and she complained of suffocation with lack of oxygen, she was immediately shifted to the intensive care unit, and treated with heavy steroids and antihypertensive along with other supportive treatment. Her liver function test (LFT) and KFT were normal, but her symptoms were not improving as expected, and she went into labor. She delivered preterm twins of $1.2 \mathrm{~kg}$ and $905 \mathrm{gm}$, there was no postpartum hemorrhage and the uterus was well contracted, she was asymptomatic till the next morning when she had slightly more than normal bleed and had not passed urine. She was catheterized and $100 \mathrm{~mL}$ of urine was collected. Ultrasound was done to rule out any placental tissue in the uterine cavity. In another 2 hours, she again started getting restless and became breathless, her x-ray chest, electrocardiogram, echocardiogram were normal, but the blood investigations showed raised liver enzymes, deranged KFT with raised creatinine, and decreased hemoglobin and platelet count. Schistocytes were present in the blood indicating hemolysis. She was diagnosed as HUS and was treated with plasma exchange, dialysis, and other supportive treatment and later transferred to the nephrology unit. She recovered completely before being discharged.

\section{Case 3}

A 30-year-old primigravida was referred with eclampsia, anuria, and altered sensorium after cesarean section for abruptio placentae. She was normotensive in her earlier antenatal visits but had developed sudden rise of blood pressure and headache for which she was taken to the hospital where she had a cesarean section for abruptio placentae.

The patient had convulsions and deranged LFT and KFT. She was intubated and treated with antihypertensive, dialysis, and other supportive treatment. She developed partial loss of vision in the left eye during the course of recovery.

Her magnetic resonance imaging showed changes in the parieto-occipital region of the brain and she was diagnosed to have postpartum eclampsia with acute kidney injury and PRES. She recovered completely with treatment and was discharged under satisfactory condition.

The following investigations are required to diagnose as well as differentiate between TTP, HUS, PRES, and HELLP.

- Complete blood count with platelets, peripheral blood smear, reticulocyte count, direct antiglobulin (Coomb's test) prothrombin time, activated partial thromboplastin time, and fibrinogen levels.

- LFT, KFT and lactate dehydrogenase (LDH).

- Fragmented RBC on blood smear is indicative of microangiopathic hemolysis, (schistocytes, helmet cells, and distorted appearing RBC).

- Evidence of hemolysis (falling hemoglobin level, polychromasia, elevated reticulocyte count, elevated serum LDH and bilirubin, and reduced haptoglobin).

- A disintegrin-like and metalloproteinase with thrombospondin type I motif 13 (ADAMTS-13) activity level-level less than $10 \%$, with the presence of antibody against ADAMTS-13 is characteristic of most TTP.

- Stool testing: Shiga toxin enzyme-linked immunosorbent assay or specific culture media for Escherichia coli. $^{1}$

\section{DISCUSSION}

The TMA is defined by the occurrence of fibrin and/ or platelet thrombi in the microvasculature resulting in mechanical hemolysis and thrombocytopenia during the antepartum or postpartum period. The two prominent causes for this are:

1. ADAMTS-13, a Von Willebrand factor (VWF) processing enzyme deficiency has emerged as a cause of a peculiar type of TMA with profound thrombocytopenia and minor renal involvement-TTP.

2. Mutations in one or more genes coding for proteins involved in regulation or activation of the alternative pathway of complement have been established as a risk factor for another type of TMA with predominant renal involvement (atypical HUS), a non-shiga toxinrelated HUS.

Pregnancy may trigger the onset or the subsequent relapses of ADAMTS-13 deficiency-related TTP as well as complement dysregulation-associated atypical HUS; complement dysregulation is also associated with HELLP syndrome, which shares several features of P-TMA. ${ }^{2}$ Absent or severe depletion of Von Willebrand cleaving 
protease ADAMTS-13, an enzyme, leads to accumulation of large VWF multimers causing microangiopathic thrombosis and severe thrombocytopenia with resultant hemolytic anemia and thrombotic syndrome. ${ }^{3}$

The HUS is a clinical syndrome characterized by progressive renal failure that is associated with microangiopathic (nonimmune Coomb's negative) hemolytic anemia and thrombocytopenia. The TTP and HUS have different causes but share many common features which include similar pathological changes, such as microangiopathic hemolytic anemia, thrombocytopenia, and neurological or renal abnormalities. Gasser et al first described HUS in 1955. ${ }^{4}$ Wardle described HUS and TTP as distinct entities. Damage to the endothelial cells is the primary event in the pathogenesis of HUS-arteriolar and capillary microthrombi and Red cell fragmentation are the cardinal lesions. The HUS is classified into two main categories depending upon its association with or without shiga-like toxin. Shiga-like toxin is not only associated with shigella dysentery, but is also elaborated by E. coli.

Various triggers for nonshiga-like toxin HUS are nonenteric infections, drugs, viruses, pregnancies, and underlying antiphospholipid syndrome and systemic lupus erythematous. ${ }^{4}$ The HUS of pregnancy most commonly occurs in primiparous females after an otherwise uncomplicated gestation and delivery and is characterized by renal failure, thrombocytopenia, and thrombotic microangiopathic hemolytic anemia in postpartum women without preeclampsia, hemorrhagic shock, disseminated intravascular coagulation (DIC), or sepsis. With plasmapheresis, 80 to $90 \%$ of patients survive an acute episode of HUS. ${ }^{5}$ Pregnancy-associated HUS occasionally develops as a complication of preeclampsia and patients may develop a full-blown HELLP syndrome. Postpartum HUS usually occurs within 3 months of delivery, the prognosis is poor with 50 to $60 \%$ mortality rate or residual renal dysfunction and hypertension in most patients. ${ }^{4}$ Although TTP and HUS are uncommon, they are clinically important because of substantial morbidity and mortality. Both TTP and HUS have been hypothesized to be as a result of platelet aggregating agents in the circulation and/or endothelial cell injury. ${ }^{6}$ The TTP that occurs during pregnancy carries the risk of relapse after delivery as well as in subsequent pregnancies. Relapses are common among those with congenital ADAMTS-13 deficiency. ${ }^{7}$

\section{MANAGEMENT}

Thrombotic microangiopathy leading to severe thrombocytopenia with resultant hemolytic anemia and thrombotic sequelae are common features of TTP, HUS, HELLP, and PRES. The treatment is slightly different for the different conditions but what remains constant is the mainstay supportive therapy with plasmapheresis and steroids.

Renal dysfunction, significantly low platelet, fever and fluctuating neurological symptoms in addition to high LDH with only moderate elevation of aspartate transaminase (AST), with elevated LDH to AST ratio and decreased ADAMTS-13 suggest the diagnosis of TTP.

The TTP-HUS syndrome occurs more commonly in women and more so is associated with pregnancy. The period during pregnancy with greatest risk for the development of TTP-HUS syndrome is near term and during postpartum. Other thrombotic events and pregnancyassociated syndromes like PET, eclampsia, and HELLP also occur during the same duration cause of the hypercoagulable state of pregnancy. The PET and progressive decreasing concentration of ADAMTS-13 may combine to increase the risk of TTP-HUS. ${ }^{3}$

Patients with less than $10 \%$ of ADAMTS-13 level and TTP respond well to plasma exchange and immunosuppression. Patients with higher levels of ADAMTS-13 and no antibody against ADAMTS-13 are unlikely to respond and should be assessed for treatment with complement inhibition. ${ }^{1}$

Patients with TTP respond to plasma exchange and corticosteroid; untreated TTP is almost always fatal. With plasma exchange, more than $85 \%$ recover and exchange has to be continued till the evidence of disease activity has subsided, which is indicated by a normal platelet count. This whole process may take from several days to weeks together. ${ }^{1}$

Severe thrombocytopenia in HELLP syndrome usually responds to steroids within 8 to 12 hours, hence, lack of this response favors TTP. The HELLP is usually associated with PET; however, PET and HELLP occur concurrently in $17 \%$ TTP. $^{8}$

In case of doubt between HELLP and TTP after delivery, plasma therapy should be started if:

- Severe thrombocytopenia and microangiopathic hemolytic anemia progress for more than 3 days following delivery.

- There are normal coagulation parameters, or resolving DIC.

- There are mental status abnormalities (such as confusion, disorientation, stupor, and coma).

- There are focal (neurologic) abnormalities (such as aphasia, dysarthria, and focal motor deficits).

- There are seizures (in association with progressive postpartum thrombocytopenia and microangiopathic hemolytic anemia).

- There is oliguric acute renal failure (in association with progressive postpartum thrombocytopenia and microangiopathic hemolytic anemia). ${ }^{7}$ 
Platelets are to be used only to prevent life-threatening hemorrhage, as it can cause increased formation of microthrombi.

Large doses of aspirin and heparin can be used in patients with prior TTP. High-dose corticosteroids and other immunosuppressive treatment are required with severe TTP. ${ }^{8}$

Patients with HUS refractory to plasma exchange and/or corticosteroids and worsening renal insufficiency will require treatment with complement inhibition with eculizumb which can sometimes reverse renal insufficiency. ${ }^{1}$ Pregnancy in patients with complement abnormalities was complicated with fetal loss and preeclampsia in 4.8 and $7 \%$ respectively. ${ }^{2}$

\section{CONCLUSION}

The TTP, HUS, HELLP, and PRES all present usually late in pregnancy or postpartum, and are known to cause mortality or severe morbidity with residual renal dysfunction. All of them may present with preexisting hypertension, PET, or eclampsia. Their investigations and treatment may have many things in common, with most of them requiring plasma exchange. Treatment is a multidisciplinary approach and with patients requiring plasma exchange and renal dialysis due to renal failure, they are best treated by nephrologists. Careful surveillance with an early diagnosis is a must for good maternal and fetal outcome.

\section{REFERENCES}

1. Kuter, DJ. Thrombotic Thrombocytopenic Purpura (TTP) and Haemolytic Uremic Syndrome(HUS). Kenilworth(NJ): Merck\& Co. Inc.; 2017.

2. Fakhouri F, Roumenina L, ProvotF, Sallée M, Caillard S, Couzi L, Essig M, Ribes D, Dragon-Durey MA, Bridoux F, et al. Pregnancy-associated haemolytic uremic syndrome revisited in the era of complement gene mutations. J Am Soc Nephrol 2010 May;21(5):859-867.

3. George JN. The association of pregnancy with thrombotic thrombocytopenic purpura-haemolytic uremic syndrome. Curr Opin Hematol 2003 Sep;10(5):339-344.

4. Parmar, MS. Hemolytic-uremic syndrome. Medscape. 2015. [cited 2016 Sep 19]. Available from: http://emedicine.medscape.com/article/201181-overview.

5. Christakos, P.; Lopez, I.; Triulzi, D. Final diagnosis-haemolytic uremic syndrome of pregnancy. Pittsburgh (PA): UPMC.

6. Elliott MA, Nichols WL. Thrombotic thrombocytopenic purpura and haemolytic uremic syndrome. Mayo Clin Proc 2001 Nov;76(11):1154-1162.

7. Wessel CH, Andreescu CE, Rombout-De Weerd S, Levin MD. Postpartum microangiopathic disorders: a case report and review of literature. Case Rep Womens Health 2014 Jul-Dec; 3-4:3-6.

8. Fyfe-Brown A, Clarke G, Nerenberg K, Chandra S, Jain V. Management of pregnancy associated thrombotic thrombocytopenic purpura. AJP Rep 2013 May;3(1):45-50. 